(2) Open Access Full Text Article

\title{
Supratherapeutic dose evaluation and effect of lesinurad on cardiac repolarization: a thorough QT/QTc study
}

\author{
This article was published in the following Dove Press journal: \\ Drug Design, Development and Therapy \\ 27 October 2016 \\ Number of times this article has been viewed
}

\section{Zancong Shen' \\ Michael Gillen ${ }^{2}$ \\ Kathy Tieu' \\ Mai Nguyen' \\ Erin Harmon' \\ David MWilson' \\ Bradley Kerr' \\ Caroline A Lee'}

'Ardea Biosciences, Inc., San Diego, CA, ${ }^{2}$ AstraZeneca LP, Gaithersburg, MD, USA
Correspondence: Zancong Shen Translational Sciences, Ardea Biosciences, Inc., 9390 Towne Centre Drive, San Diego, CA, USA

Tel + I 8586526623

Email zshen@ardeabio.com
Introduction: Lesinurad is a selective uric acid reabsorption inhibitor approved in the United States and Europe for treatment of gout in combination with a xanthine oxidase inhibitor. A maximum tolerated dose study was conducted to determine the lesinurad supratherapeutic dose, followed by a thorough QTc study to characterize the effect of lesinurad on cardiac repolarization.

Methods: The maximum tolerated dose study was a randomized, double-blind, placebo-controlled, single-ascending dose study that enrolled 35 healthy men and women. Lesinurad plasma exposure (maximum observed plasma concentration and area under the plasma concentration versus time curve) was determined at doses of $800 \mathrm{mg}, 1,200 \mathrm{mg}$, and 1,600 mg. The thorough QTc study was a double-blind, four-period, placebo-controlled crossover study with 54 healthy men and women who received single doses of lesinurad 1,600 mg (supratherapeutic dose), lesinurad $400 \mathrm{mg}$, moxifloxacin $400 \mathrm{mg}$, and placebo in randomized sequence. Digital 12-lead electrocardiograms were recorded at eleven time points over 24 hours in each treatment period. QT intervals were corrected for heart rate using an individual-specific correction factor (QTcI).

Results: The upper bound of the one-sided $95 \%$ confidence interval for time-matched, placebosubtracted, baseline-adjusted QTcI intervals $(\Delta \Delta \mathrm{QTcI})$ was $<10 \mathrm{~ms}$ for both the lesinurad $400 \mathrm{mg}$ and 1,600 mg doses. $\Delta \Delta \mathrm{QTcI}$ was independent of lesinurad concentrations. No QTcI thresholds $>480 \mathrm{~ms}$ or QTcI increases $>30 \mathrm{~ms}$ were observed. Moxifloxacin mean QTcI intervals were $>5 \mathrm{~ms}$, and the lower bounds of the $90 \%$ confidence interval were $>5 \mathrm{~ms}$ at 2 hours, 3 hours, and 4 hours, confirming assay sensitivity.

Conclusion: Lesinurad, at supratherapeutic doses, does not have a significant effect on the QT interval in healthy male or female subjects.

Keywords: pharmacokinetics, maximum tolerated dose study, QT interval, selective uric acid reabsorption inhibitor

\section{Introduction}

Gout is the most common form of inflammatory arthritis. ${ }^{1}$ It is caused by hyperuricemia (defined typically as serum uric acid [sUA] $>6.8 \mathrm{mg} / \mathrm{dL}$ ) that is mainly caused due to inefficient uric acid excretion. ${ }^{2,3}$ Hyperuricemia can lead to urate crystal deposition disease. The deposition of monosodium urate crystals in musculoskeletal structures, kidneys, and other tissues causes chronic inflammation, acute gout flares, and potentially chronic arthritis with joint damage and disfiguring tophi, kidney stones, and chronic kidney disease.

Gout requires chronic lifestyle management and pharmacological treatment to maintain effective control of sUA levels. ${ }^{4-6}$ Given that patients with gout will need 
continued therapy, investigational drugs require rigorous evaluation for potential long-term safety concerns, including potential effects on cardiac repolarization. Delayed ventricular repolarization, as measured by a prolonged QT interval, has been associated with an increased risk of arrhythmias, including torsades de pointes. $^{7}$

Lesinurad is a selective uric acid reabsorption inhibitor approved in the United States and Europe for use in combination with a xanthine oxidase inhibitor for the chronic treatment of hyperuricemia associated with gout in patients who have not achieved target sUA levels with a xanthine oxidase inhibitor alone. Lesinurad inhibits the uric acid transporter URAT1 in the proximal tubules of the kidney, ${ }^{8}$ resulting in increased urinary excretion of uric acid and decrease in sUA. ${ }^{9}{ }^{10}$ Phase I and II trials conducted for lesinurad have used doses up to $600 \mathrm{mg} / \mathrm{d}$ in once-daily multiple doses in either healthy volunteers or patients with hyperuricemia and gout. No signal of a likely electrocardiogram (ECG) effect was noted in these studies.

Current regulatory recommendations are that all nonantiarrhythmic compounds undergo clinical evaluation of QT/QTc interval prolongation by means of a thorough QT (TQT) study. ${ }^{7}$ The purpose of this study was to first select a supratherapeutic dose of lesinurad in a maximum tolerated dose (MTD) study and then to include that dose in a TQT study to assess the potential for a single dose of lesinurad to prolong ventricular repolarization by testing the hypothesis that lesinurad does not prolong the QTc interval. The validity of the conclusion was assessed by the ability of a positive control, moxifloxacin, to increase the QTc interval.

\section{Methods}

This study was designed and monitored in accordance with the ethical principles of Good Clinical Practice as defined by the International Conference on Harmonisation and the Declaration of Helsinki. The Alpha Independent Review Board approved the protocol before trial commencement and all subjects gave written, informed consent.

\section{Subjects}

Healthy male or female subjects between the age of 18 years and 50 years with a body mass index between $19 \mathrm{~kg} / \mathrm{m}^{2}$ and $28 \mathrm{~kg} / \mathrm{m}^{2}$ were included. Female subjects of childbearing potential who were nonpregnant and nonlactating and used a nonhormonal double barrier method of birth control were included in this study. Subjects were to be homozygous CYP2C9 wild-type $(* 1 / * 1)$ "normal metabolizers" or heterozygous $(* 1 / * 2, * 1 / * 3, * 1 / * 4, * 1 / * 5, * 1 / * 6, * 1 / * 8$, or *1/*11) “intermediate metabolizers". Subjects who had two or more detectable polymorphisms in CYP2C9 were excluded from the MTD and TQT studies. Subjects of Asian, African, and African-American ethnicity were excluded from the MTD study as a safety precaution since these races are known to sometimes express the CYP2C9 polymorphisms *4 and $* 8$, which were not screened for in the MTD study. Subjects of Asian, African, and African-American ethnicity were allowed in the TQT study since screening was performed for the CYP2C9 polymorphisms *4 and *8.

Exclusion criteria included an abnormal screening ECG indicating a second- or third-degree atrioventricular block or one or more of the following: QRS $>110 \mathrm{~ms}$, QTc $>450 \mathrm{~ms}$, PR interval $>210 \mathrm{~ms}$, or any rhythm other than sinus rhythm, which was interpreted by the investigator to be clinically significant. Subjects also were excluded if they had a history of risk factors for torsades de pointes, including unexplained syncope, known long QT syndrome, heart failure, myocardial infarction, angina, or clinically significant abnormal laboratory assessments including hypokalemia, hypercalcemia, and hypomagnesemia. Those with a family history of long QT syndrome or Brugada syndrome were also excluded.

\section{Study design}

The MTD study, conducted at Comprehensive Clinical Development (Tacoma, WA, USA, and Miramar, FL, USA), was a randomized, double-blind, placebo-controlled, single-ascending dose study that assessed the safety, tolerability, pharmacokinetics (PK), and pharmacodynamics of lesinurad in healthy adult male and female subjects. The planned supratherapeutic doses for each group were lesinurad $800 \mathrm{mg}, 1,200 \mathrm{mg}, 1,600 \mathrm{mg}$, and 2,000 mg. Subjects in each dose group were randomized 5:1 to lesinurad (ten subjects [five male and five female]) or placebo (two subjects [one male and one female]) with 2-7 days between dosing of each group. Dose escalation did not proceed if the next planned dose was projected to achieve maximum observed plasma concentration $\left(C_{\max }\right)$ or area under the plasma concentration versus time curve (AUC) that exceeded the no observable adverse effect level (NOAEL) exposures in preclinical toxicology studies. Advancement to the next dose level was to be stopped if two or more subjects reported a serious adverse event (AE) or experienced a severe AE that was considered to be possibly related to study medication, occurring at the current dose level. 
The TQT study, conducted at Comprehensive Clinical Development (Miramar, FL, USA), was a blinded (except for moxifloxacin), randomized, four-arm crossover study in healthy male and female subjects. There were four single-dose treatment periods, with a minimum 5-day washout period between each dose. Treatment sequences were randomized based on the Williams Latin Square design for a crossover study. Each subject received the following four treatments, administered in the order prescribed by the sequence to which the subject had been randomly assigned: lesinurad supratherapeutic dose (1,600 mg), lesinurad $400 \mathrm{mg}$, overencapsulated moxifloxacin $400 \mathrm{mg}$, and placebo. Moxifloxacin was included as a positive control to determine the assay sensitivity. Subjects fasted for at least 10 hours and then consumed a standard breakfast (at least $643 \mathrm{kcal}$ and at least 23\% fat) within 30 minutes before dosing. Lesinurad, matching placebo tablets, and moxifloxacin tablets were taken orally with $\sim 240 \mathrm{~mL}$ of water.

\section{Safety}

Vital signs, 12-lead safety ECG, physical examinations, and safety laboratory analyses involving routine hematology, serum chemistry, and urinalysis were obtained throughout the study, at a follow-up examination on day 5 in the MTD study, and $\sim 1$ week after the last treatment period in the TQT study. All AEs were evaluated by the investigator and characterized with respect to intensity, duration, relationship to study drug, and outcome.

\section{Pharmacokinetics assessment}

Blood samples were collected in the MTD study at predose and at 0.5 hour, 1 hour, 1.5 hours, 2 hours, 3 hours, 4 hours, 5 hours, 6 hours, 8 hours, 10 hours, 12 hours, 16 hours, 24 hours, 30 hours, 36 hours, 48 hours, 60 hours, 72 hours, 86 hours, and 96 hours and in the TQT study at predose, 1 hour, 2 hours, 3 hours, 4 hours, 5 hours, 6 hours, 8 hours, 10 hours, 12 hours, 16 hours, and 23 hours. Plasma samples were analyzed for lesinurad by Ardea Biosciences, Inc. (San Diego, CA, USA). The samples were prepared by protein precipitation and were quantified using highperformance liquid chromatography with tandem mass spectroscopy. PK parameters for lesinurad were calculated using noncompartmental analysis. Plasma PK parameters included $C_{\max }$, time to $C_{\max }\left(\mathrm{T}_{\max }\right)$, AUC from 0-23 hours (AUC [0-23], AUC from 0-24 hours (AUC [0-24], AUC from 0-96 hours (AUC [0-96]). AUC was calculated using the linear trapezoidal rule.

\section{Continuous 12-lead electrocardiograms}

ECGs were obtained digitally using a Mortara Instrument (Mortara, Milwaukee, WI, USA) H-12+ ECG continuous 12-lead digital recorder. In the MTD study, continuous 12-lead ECGs were obtained beginning prior to dosing on day 1 through 23 hours postdose for safety purposes only. In the TQT study, continuous 12-lead ECGs were obtained on day -1 (baseline) and on day 1 of each treatment period. ECGs were obtained as four 12-lead ECGs, which were downloaded from the $\mathrm{H}-12+$ flash card $~ 1$ minute apart at baseline, and again on day 1 at the following time points for all four treatment periods: 1 hour, 2 hours, 3 hours, 4 hours, 5 hours, 6 hours, 8 hours, 10 hours, 12 hours, 16 hours, and 23 hours postdose. Continuous records of ECGs were used for the end point analysis.

\section{Electrocardiogram analysis - TQT study} ECGs were analyzed by eResearch Technology, Inc. (Philadelphia, PA, USA) using their validated data management system, EXPERT. Interval duration measurements were collected using computer-assisted caliper placements on three consecutive beats. On-screen measurements of the RR, PR, QRS, and QT interval durations were performed and the derived variables QT interval corrected using Fridericia's formula (QTcF), QT interval corrected for heart rate (HR) using Bazett's formula (QTcB), and HR were obtained. Each fiducial point (onset of QRS, offset of $\mathrm{T}$ wave, etc) was electronically marked.

The primary variable was QT interval corrected using an individual-specific interval correction for heart rate (QTcI), which was derived from the ECG using an individual-specific correction factor. The goal was to find $\beta$ such that QTcI is a constant, where $\mathrm{QTcI}=\mathrm{QT} /(\mathrm{RR})^{\beta}$, with the $\mathrm{QT}$ interval expressed in milliseconds and the RR interval in seconds. The equation can be rewritten as $\log (\mathrm{QT})=\alpha+\beta \times \log (\mathrm{RR})$. The exponent estimate was obtained by numerical iteration such that slope for the QT-RR relationship was closest to zero or using regression analysis on log-transformed data based on the least squares approach.

\section{Safety assessment}

Safety assessments included AEs based on the medical review of $\mathrm{AE}$ reports and the results of vital sign measurements, 12-lead ECGs, physical examinations, and clinical laboratory tests. AEs were categorized according to the Medical Dictionary for Regulatory Activities and were reviewed for potential significance and clinical importance 
by the study site investigator. Serious AEs were defined as AEs that resulted in death; hospitalization or prolongation of existing hospitalization; persistent or significant disability or congenital anomaly; were life-threatening; or were considered an important medical event.

\section{Statistical analyses \\ Populations}

The safety population consisted of all subjects who received at least one dose of lesinurad, moxifloxacin, or placebo. The PK population consisted of all subjects who received at least one dose of lesinurad and had evaluable PK data. The ECG analysis was performed on all subjects in the TQT study who were randomized, received at least one dose of study medication, and had at least one baseline ECG and at least one on-treatment, postdose, time-matched ECG within the same treatment period.

\section{Pharmacokinetics statistics}

Descriptive summary statistics were grouped according to the dose group, by separate sexes as well as combined sexes. All descriptive statistics calculations were performed using validated WinNonlin Professional, Version 5.2 (Pharsight Corporation, Mountain View, CA, USA). Dose linearity was assessed using linear regression analysis.

Geometric mean ratios of plasma exposure between dose levels were assessed by comparing natural log-transformed PK parameters $\left(C_{\max }, \mathrm{AUC}_{[0-\text { inf }]}\right)$. Estimates of geometric least squares means and geometric mean ratios with the corresponding $90 \% \mathrm{CI}$ (confidence interval) were performed by statistical tests in the bioequivalence wizard tool in WinNonlin Professional, Version 5.2 (Pharsight Corporation).

\section{Electrocardiography}

The primary ECG end point in the TQT study was the timematched change from baseline in QTc, placebo corrected, based on an optimized interval correction for HR (QTcI) method $(\Delta \Delta \mathrm{QTCI})$. The secondary ECG end points were QTcF and QTcB (for historic reasons), HR, PR interval, uncorrected QT interval, change in ECG morphological patterns, and correlation between the QTcI change from baseline and plasma concentrations of lesinurad. To test for assay sensitivity, a linear contrast comparing moxifloxacin versus placebo from the primary analysis model on mean QTcI over 1 hour, 2 hours, 3 hours, 4 hours, and 5 hours after dosing was analyzed. At least one time point needed to be demonstrated where the lower confidence bound of the mean difference of moxifloxacin and placebo was $>5 \mathrm{~ms}$.

\section{QT versus lesinurad concentration}

\section{analysis}

The QT versus lesinurad concentration analysis used a linear mixed-effect model. Plasma concentration, intercept, and subject were included as random effects. In this analysis, the $\Delta \Delta$ refers to the subtracted difference of drug-placebo change from the mean values before dosing at each time point after dosing. This model was used to estimate the population slope and the standard error of the slope of the relationship between the placebo-adjusted/corrected change from baseline in QTc intervals (QTcI, QTcF, and QTcB) and plasma concentrations of lesinurad. If this model did not converge, then plasma concentration was included as a fixed effect with intercept and subject included as random effects. If the $P$-value of the slope was $<0.05$, then a linear relationship was declared. The mean maximum effect as well as the upper one-sided $95 \%$ CI was calculated.

\section{Sample size}

Formal power calculations were not performed for the sample size in the MTD study. A sample size of ten subjects per dose level was chosen to provide an adequate number of healthy subjects for which a clinical determination of tolerability could be made. Assuming a drug effect of $2 \mathrm{~ms}$ and an SD of $10 \mathrm{~ms}$ in the TQT study, a sample size of 44 evaluable subjects per group was required for at least $80 \%$ power to show that the upper limit of the $90 \%$ CI (two-sided) for the comparison of lesinurad to placebo would fall below $10 \mathrm{~ms}$.

\section{Results \\ Subject disposition and baseline characteristics}

A total of 89 subjects were dosed, 35 (18 males, 17 females) in the MTD study and 54 (28 males, 26 females) in the TQT study. All subjects in the MTD study completed the study while two subjects in the TQT study were withdrawn. One withdrew consent and the other was lost to follow-up. Demographic characteristics of each group at screening are shown in Table 1. The mean body weight and body mass index were broadly similar for subjects in both studies.

\section{MTD study}

The geometric mean PK parameters for lesinurad following a single $800 \mathrm{mg}, 1,200 \mathrm{mg}$, or 1,600 $\mathrm{mg}$ dose are summarized in Table 2. Median $\mathrm{T}_{\max }$ was 5 hours across dose levels, while $C_{\max }$ and $\mathrm{AUC}_{[0-\mathrm{inf}]}$ increased with increasing dose level. In order to extrapolate linearity across the range of doses studied with lesinurad (200-1,600 mg), additional data from two other 
Table I Demographic characteristics at screening - MTD and TQT studies

\begin{tabular}{|c|c|c|c|c|c|}
\hline & \multicolumn{4}{|l|}{ MTD study } & \multirow{2}{*}{$\begin{array}{l}\text { TQT study } \\
(\mathrm{N}=54)\end{array}$} \\
\hline & $\begin{array}{l}\text { Pooled placebo } \\
(n=6)\end{array}$ & $\begin{array}{l}\text { Lesinurad } 800 \mathrm{mg} \\
(\mathrm{n}=9)\end{array}$ & $\begin{array}{l}\text { Lesinurad I,200 mg } \\
(n=10)\end{array}$ & $\begin{array}{l}\text { Lesinurad } 1,600 \mathrm{mg} \\
(\mathrm{n}=10)\end{array}$ & \\
\hline Age, years, mean (SD) & $36(10.4)$ & $26(8.0)$ & $31(8.0)$ & $42(7.0)$ & $35(8.4)$ \\
\hline Body weight, kg, mean (SD) & $73.5(8.8)$ & 70.4 (II.7) & $74.8(10.7)$ & $67.7(14.0)$ & $68.9(9.4)$ \\
\hline BMI, kg/m², mean (SD) & $25.6(1.9)$ & $23.8(3.1)$ & $24.7(2.4)$ & $24.5(2.4)$ & $25.3(1.9)$ \\
\hline \multicolumn{6}{|l|}{ Sex, n (\%) } \\
\hline Male & $3(50)$ & $5(56)$ & $5(50)$ & $5(50)$ & $28(52)$ \\
\hline Female & $3(50)$ & $4(44)$ & $5(50)$ & $5(50)$ & $26(48)$ \\
\hline \multicolumn{6}{|l|}{ Race, n (\%) } \\
\hline White & $6(100)$ & $9(100)$ & $10(100)$ & $10(100)$ & $54(100)$ \\
\hline \multicolumn{6}{|l|}{ Ethnicity, n (\%) } \\
\hline Hispanic or Latino & $2(33.3)$ & I (II.I) & 0 & $8(80)$ & $54(100)$ \\
\hline Not Hispanic or Latino & $4(66.7)$ & 8 (88.9) & $10(100)$ & $2(20)$ & 0 \\
\hline
\end{tabular}

Abbreviations: BMI, body mass index; MTD, maximum tolerated dose; TQT, thorough QT.

Phase I studies (RDEA594-105 and RDEA594-109) were included (Figure 1). The average $C_{\max }$ and $\mathrm{AUC}_{[0-\text { inf }]}$ exposures were $\sim 20 \%-40 \%$ higher in female subjects at each dose level. Lesinurad $C_{\max }$ increased linearly with increased dose levels in both male and female subjects, while $\mathrm{AUC}_{[0-\text { inf }]}$ was linear up to $1,200 \mathrm{mg}$ with nonlinear increases at $1,600 \mathrm{mg}$ in both male and female subjects. The $\mathrm{AUC}_{[0-\mathrm{inf}]}$ for lesinurad $1,600 \mathrm{mg}$ was 6.4-fold and 6.2-fold greater in male and female subjects, respectively, than for lesinurad $400 \mathrm{mg}$.

Nonclinical toxicology data from rats and monkeys provided safety margins to support a 2,000 mg dose in humans. However, the 2,000 mg planned dose was not conducted due to the greater than expected exposure at the $1,600 \mathrm{mg}$ dose, which predicted that the $2,000 \mathrm{mg}$ dose would exceed the NOAEL exposure limit. The supratherapeutic dose used for the TQT study was therefore $1,600 \mathrm{mg}$.

\section{TQT study}

\section{QTc interval}

Lesinurad $400 \mathrm{mg}$ was chosen as the therapeutic dose as it was planned to be used in Phase III clinical trials.
The placebo-corrected change from baseline in QTcI duration for the lesinurad doses and moxifloxacin at each time point is presented in Figure 2. The placebo-subtracted, baselineadjusted mean QTcI for lesinurad (400 mg and 1,600 mg) was $<5 \mathrm{~ms}$ at each time point, and all upper bounds of the two-sided $90 \% \mathrm{CI}$ for the contrasts were $<10 \mathrm{~ms}$. The maximum placebo-subtracted, baseline-adjusted mean effect of lesinurad $400 \mathrm{mg}$ for QTcI was $3.1 \mathrm{~ms}$ at 3 hours after dosing (90\% CI, 0.8-5.3). For lesinurad 1,600 mg, the maximum placebo-corrected, baseline-adjusted mean effect for QTcI was $4.0 \mathrm{~ms}$ at 6 hours after dosing (90\% CI, 1.7-6.2). There were no QTcI thresholds $>480 \mathrm{~ms}$ or increases from baseline $>30 \mathrm{~ms}$ for QTcI over the 24-hour period following lesinurad, placebo, or moxifloxacin administration. One subject each in the lesinurad 1,600 $\mathrm{mg}$ and moxifloxacin groups had an increase in QTcF or QTcB >30 ms.

For moxifloxacin, with the exception of the contrast at 0.5 hour and 12 hours after dosing, all point estimates for the contrasts between moxifloxacin $400 \mathrm{mg}$ and placebo for time-matched QTcI were $>5 \mathrm{~ms}$, and the lower bounds of the $90 \%$ CI were $>5 \mathrm{~ms}$ at hours 2,3 , and 4 of the five preselected

Table 2 Plasma pharmacokinetics of lesinurad following a single dose of lesinurad $800 \mathrm{mg}, \mathrm{I}, 200 \mathrm{mg}$, or I,600 mg to healthy male and female subjects - MTD and TQT study

\begin{tabular}{|c|c|c|c|c|c|c|}
\hline \multirow[t]{2}{*}{ Dose (mg) } & \multirow[t]{2}{*}{$\mathbf{N}$} & Median (range) & \multicolumn{4}{|c|}{ Geometric mean $(95 \% \mathrm{CI})$} \\
\hline & & $\mathbf{T}_{\max }{ }^{a}(\mathbf{h})$ & $C_{\max }(\mu g / m L)$ & $\operatorname{AUC}_{[0-24]}(\mu \mathrm{g} \cdot \mathrm{h} / \mathrm{mL})$ & $\operatorname{AUC}_{[0-96]}(\mu \mathrm{g} \cdot \mathrm{h} / \mathrm{mL})$ & $\operatorname{AUC}_{[0-\mathrm{inf}]}(\mu \mathrm{g} \cdot \mathbf{h} / \mathrm{mL})$ \\
\hline \multicolumn{7}{|l|}{ MTD study } \\
\hline 800 & 9 & $5.00(1.50-5.00)$ & $34.8(27.4-44.1)$ & 132 (103-167) & $133(105-170)$ & $134(105-170)$ \\
\hline $\mathrm{I}, 200$ & 10 & $5.00(2.00-6.00)$ & $54.9(46.8-64.3)$ & $238(202-28 I)$ & $242(205-286)$ & $243(206-286)$ \\
\hline 1,600 & 10 & $5.00(2.00-6.00)$ & $76.8(63.5-92.8)$ & $455(360-576)$ & $463(366-586)$ & $470(365-605)$ \\
\hline \multicolumn{7}{|l|}{ TQT study } \\
\hline 400 & 53 & $3.00(1.00-6.00)$ & $16.1(\mid 4.7-17.6)$ & $60.3(56.0-64.8)^{\mathrm{b}}$ & ND & $60.3(56.0-64.8)$ \\
\hline 1,600 & 54 & $5.00(2.00-6.00)$ & $70.2(65.6-75.1)$ & $391(362-422)^{\mathrm{b}}$ & ND & $391(362-422)$ \\
\hline
\end{tabular}

Notes: a Median (min-max). ${ }^{b} A \cup C_{[0-23]}$, area under the plasma concentration versus time curve from time $0-23$ hours.

Abbreviations: $A \cup C_{[0-24]}, A \cup C_{[0-96]}{ }^{[0-23]}, A \cup C_{[0-i n f]}$, area under the plasma concentration versus time curve from time $0-24$ hours, 96 hours, and infinity, respectively; $C l$, confidence interval; $C_{\max }$, maximum observed plasma concentration; $T_{\max }$, time of maximum observed plasma concentration; MTD, maximum tolerated dose; ND, not determined; min, minimum; max, maximum; h, hours; TQT, thorough QT. 


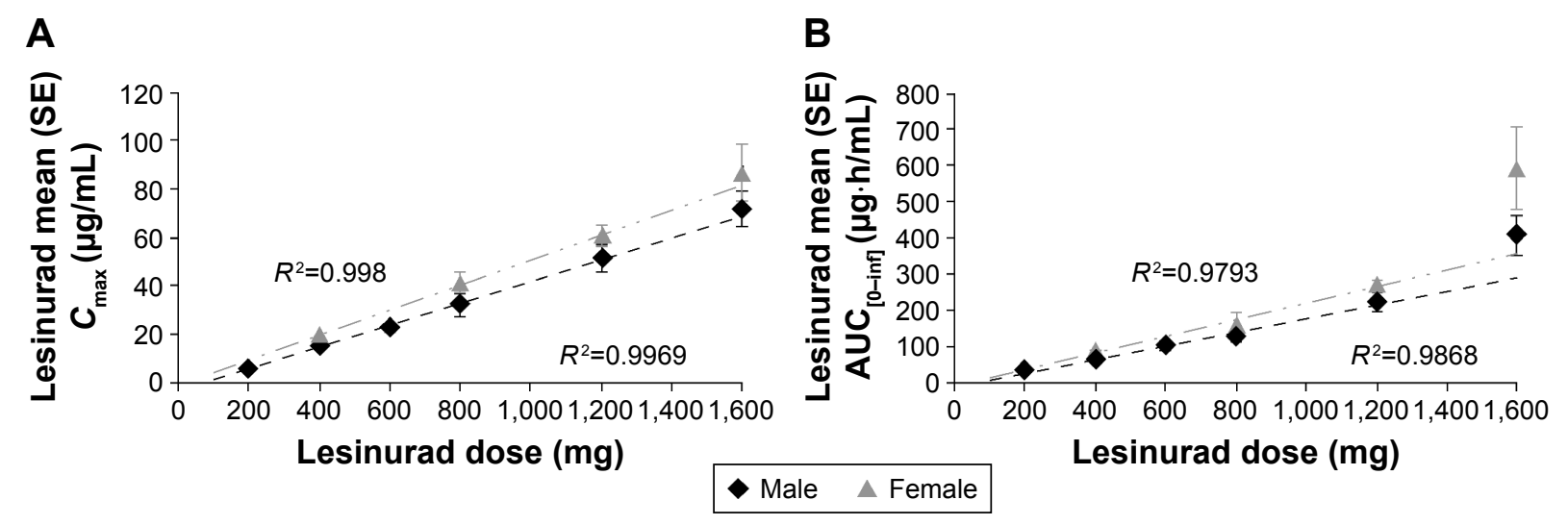

B

Figure I Dose linearity assessment of $C_{\max }(\mathbf{A})$ and $A \cup C_{[0-i n f]}(\mathbf{B})$ for lesinurad in males and females - MTD study.

Notes: The $200 \mathrm{mg}, 400 \mathrm{mg}$, and $600 \mathrm{mg}$ data for males were taken from study RDEA594-109, while the 400 mg data for females were from study RDEA594-I05.

Abbreviations: $A \cup C_{[0-i n f}$, area under the plasma concentration-time curve from zero to infinity; $C_{\text {max }}$, maximum observed plasma concentration; MTD, maximum tolerated dose; SE, standard error.

time points, satisfying assay sensitivity for the trial. The maximum placebo-corrected, baseline-adjusted mean effect of moxifloxacin $400 \mathrm{mg}$ for QTcI was $10.0 \mathrm{~ms}$ at 3 hours after dosing, and the lower bound of the two-sided $90 \% \mathrm{CI}$ was $6.8 \mathrm{~ms}$. Placebo change from baseline was $-4 \mathrm{~ms}$, showing that the background QTc variability was well controlled.

No sex differences were observed for the placebo- and baseline-corrected analysis with either lesinurad dose or moxifloxacin.

\section{Concentration-QT relationship}

Mean individual QTcI intervals versus lesinurad (400 mg and $1,600 \mathrm{mg}$ ) plasma concentration are presented in Figure 3. There was no apparent concentration-dependent effect of lesinurad on QTcI. The estimated slope was essentially zero $(0.0386 \mathrm{~ms} / \mu \mathrm{g}$ per milliliter $)$, and the test of the hypothesis of zero slopes was statistically significant $(P=0.0271)$. Similar results were observed for QTcF and QTcB.



Figure 2 Placebo-corrected change from baseline QTcl versus sample time. Abbreviation: QTcl, QT interval corrected using an individual-specific interval correction for heart rate.

\section{Effect on HR and PR and QRS intervals}

The mean change from baseline placebo-corrected for HR for the lesinurad $400 \mathrm{mg}$ and $1,600 \mathrm{mg}$ doses and moxifloxacin was $-0.5 \mathrm{bpm},+0.6 \mathrm{bpm}$, and $+1.0 \mathrm{bpm}$, respectively. The corresponding change for the PR interval was $-0.1 \mathrm{~ms},-1.3 \mathrm{~ms}$, and $-2.0 \mathrm{~ms}$, respectively, and was $+0.1 \mathrm{~ms},-0.5 \mathrm{~ms}$, and $-0.5 \mathrm{~ms}$ for the QRS interval.

\section{Pharmacokinetics}

PK parameters of lesinurad are presented in Table 2. Median $\mathrm{T}_{\max }$ occurred at 3 hours following dosing with lesinurad $400 \mathrm{mg}$ and was 5 hours postdose at the $1,600 \mathrm{mg}$ dose. The $\mathrm{AUC}_{[0-\mathrm{inf}]}$ was 6.5-fold greater for lesinurad $1,600 \mathrm{mg}$ than for lesinurad $400 \mathrm{mg}$, while the increase in $C_{\max }$ appeared dose-proportional. The average $C_{\max }$ and $\mathrm{AUC}_{[0-\text { inf }]}$ exposures were $13 \%-17 \%$ higher in females than in males at each dose level, but the sex difference was minimal when they were normalized for dose and body weight (Table 3).

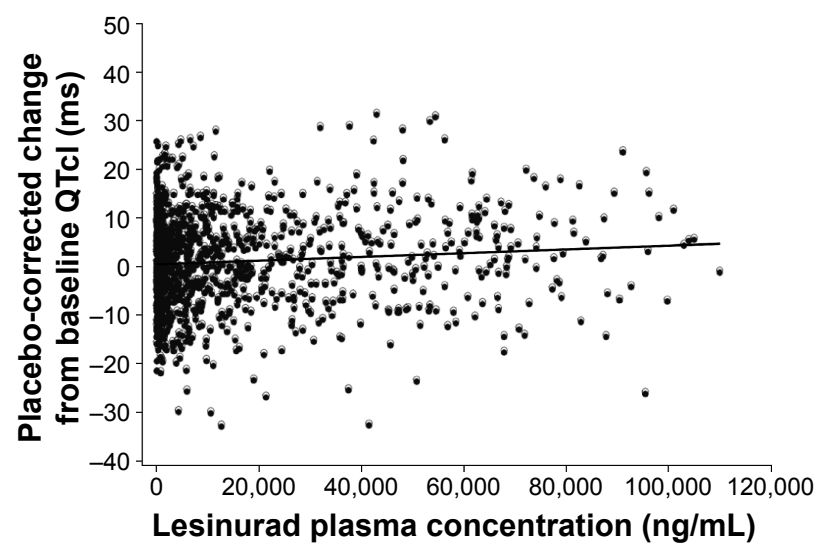

Figure $3 \mathrm{QTcl}$ placebo-corrected change from baseline versus lesinurad plasma concentration.

Abbreviation: QTcl, QT interval corrected using an individual-specific interval correction for heart rate. 
Table 3 Effect of sex on lesinurad pharmacokinetics following lesinurad dosing at $400 \mathrm{mg}$ and I,600 mg - TQT study

\begin{tabular}{|c|c|c|c|c|}
\hline \multirow[t]{2}{*}{ Dose (mg) } & \multirow[t]{2}{*}{ Parameter } & \multirow[t]{2}{*}{$\mathbf{N}$ (female/male) } & \multirow{2}{*}{$\begin{array}{l}\text { Geometric mean } \\
\text { ratio } \%(90 \% \mathrm{CI}) \\
\text { Female/male }\end{array}$} & \multirow{2}{*}{$\begin{array}{l}\text { Dose and body weight normalized } \\
\text { geometric mean ratio } \%(90 \% \mathrm{Cl}) \\
\text { Female/male }\end{array}$} \\
\hline & & & & \\
\hline \multirow[t]{2}{*}{400} & $C_{\max }$ & $25 / 28$ & II $15(99.4-134)$ & $98.4(85.3-113)$ \\
\hline & $\mathrm{AUC}_{[0-\text { inf }}$ & $25 / 28$ & II 3 (99.8-127) & $95.9(85.8-107)$ \\
\hline \multirow[t]{2}{*}{1,600} & $C_{\max }$ & $26 / 28$ & $116(104-129)$ & $99.0(88.8-110)$ \\
\hline & $A \cup C_{[0-i n f]}$ & $26 / 28$ & $116(102-132)$ & $99.5(88.1-112)$ \\
\hline
\end{tabular}

Abbreviations: $A \cup C_{[0-\text { inf }}$, area under the plasma concentration-time curve from zero to infinity; $\mathrm{Cl}$, confidence interval; $C_{\max }$, maximum observed plasma concentration; TQT, thorough QT.

\section{Safety}

In the MTD study, eight out of 29 subjects $(27.6 \%)$ who received lesinurad and no subjects who received placebo reported treatment-related AEs after dosing. AEs were reported by four subjects (44.4\%) given lesinurad $800 \mathrm{mg}$, two subjects $(20.0 \%)$ given $1,200 \mathrm{mg}$, and two subjects $(20.0 \%)$ given $1,600 \mathrm{mg}$. Only three subjects reported drug-related AEs, with mild events of headache reported by two subjects (one each given lesinurad $1,200 \mathrm{mg}$ or $1,600 \mathrm{mg}$ ) constipation by one subject given $1,600 \mathrm{mg}$. All AEs were of mild severity. Treatment-related AEs in the TQT study were reported by five subjects (9.4\%) given lesinurad $400 \mathrm{mg}$ and five subjects (9.3\%) given lesinurad $1,600 \mathrm{mg}$, nine subjects (17.0\%) given placebo, and eight subjects (14.8\%) given moxifloxacin. Most AEs were mild in intensity, except for one $\mathrm{AE}$ of moderate intensity in each of the placebo, lesinurad $400 \mathrm{mg}$, and 1,600 $\mathrm{mg}$ groups. The incidence of drug-related AEs was low, with only two subjects reporting drug-related AEs. One subject reported a mild event of headache following dosing with lesinurad $400 \mathrm{mg}$ and one subject reported mild events of nausea and pyrexia and a moderate event of headache following dosing with lesinurad 1,600 mg. One severe AE was reported; an episode of syncope $\sim 1$ day after receiving a dose of placebo in treatment period 2 that followed moxifloxacin $400 \mathrm{mg}$ in period 1. The episode was considered to be unrelated to the study drug. No deaths occurred in either study. There were no treatment or dose-related trends in the serum biochemistry (with the exception of expected decreased sUA levels), hematology, urinalysis, or vital signs in either the MTD or TQT study. There were no clinically relevant findings on analysis of the 12-lead safety ECGs for individual subjects dosed with lesinurad in either study.

\section{Discussion}

Assessment of QTc interval is required for all compounds in drug development to determine if they cause a delay in cardiac repolarization. ${ }^{7}$ The objective of the TQT study was to provide a rigorous assessment of the potential for lesinurad to prolong ventricular repolarization in human subjects at both therapeutic and supratherapeutic doses. The supratherapeutic dose of lesinurad was determined to be 1,600 $\mathrm{mg}$ in the MTD study, as the plasma exposure of a 2,000 $\mathrm{mg}$ dose was projected to exceed the NOAEL. The primary end point of the TQT study compared the change in QTc interval from predose baseline values between active and placebo treatment.

The mean QTc intervals for lesinurad evaluated at $400 \mathrm{mg}$ and 1,600 $\mathrm{mg}$ were shown not to be prolonged using an individual-specific correction method (QTcI), as all upper bounds of the $90 \%$ CI for the difference in mean QTc interval between either dose of lesinurad and placebo were $<10 \mathrm{~ms}$. A negative result in a TQT study is defined as one in which the upper bound of the $95 \%$ one-sided CI for the largest time-matched mean effect of the drug on the QTc interval excludes $10 \mathrm{~ms}$. This definition implies that the mean effect of a study drug on the QTc interval is not $>5 \mathrm{~ms} .{ }^{11}$ Both doses of lesinurad met this requirement, as the largest placebo-subtracted, baseline-adjusted mean QTcI interval for any dose was $4.0 \mathrm{~ms}$. In addition, this study incorporated concentration-QT modeling. ${ }^{11}$ The plot of QTcI versus lesinurad concentration was essentially flat, with a slope that was not significantly different from zero. The lack of an effect of lesinurad on the QTc interval is consistent with in vitro hERG data, preclinical studies, and early clinical studies.

In accordance with regulatory guidelines, moxifloxacin was included in this study as a positive control. The sensitivity of the assay to detect small increases in QTc interval was established, where the lower bound of the $90 \%$ CI for $\Delta \Delta \mathrm{QTcI}$ at one time point was $>5 \mathrm{~ms}$. The time course for the QTc effect of moxifloxacin showed that the peak effect occurred around the time of $C_{\max }{ }^{12}$ However, moxifloxacin PK samples were not analyzed in this study. Therefore, it was prospectively decided to determine the $\Delta \Delta \mathrm{QTcI}$ for moxifloxacin over each of the 1- to 5-hour time points that 
encompass the $\mathrm{T}_{\max }$ range for moxifloxacin, with the aim to demonstrate that the lower confidence bound of the mean difference of moxifloxacin and placebo was $>5 \mathrm{~ms}$ at at least one of the time points. The lower confidence bound was $>5 \mathrm{~ms}$ at hours 2,3 , and 4 . In addition, the maximum baseline-adjusted, placebo-subtracted QTcI for moxifloxacin was $10.0 \mathrm{~ms}$, and the shape of the $\Delta \Delta \mathrm{QTcI}$ versus time curve for moxifloxacin was consistent with previously published data. $^{12,13}$ Thus, assay sensitivity was rigorously confirmed and validated the results for lesinurad.

Although $400 \mathrm{mg}$ was included as the therapeutic dose, the approved clinical dose is $200 \mathrm{mg}$. Therefore, the supratherapeutic dose was eight-fold higher than the therapeutic dose and due to nondose proportional PK, the exposure was $\sim 13$-fold higher. Lesinurad is primarily metabolized by CYP2C9, with 30\%-40\% of lesinurad excreted unchanged in urine. In other studies, the moderate CYP2C9 inhibitor, fluconazole, caused approximately a $60 \%$ increase in lesinurad AUC, whereas lesinurad exposure was approximately doubled in subjects genotyped as CYP2C9 poor metabolizers (hence their exclusion from these studies). Severe renal impairment also doubled lesinurad exposure and moderate hepatic impairment increased lesinurad exposure by 33\%. Therefore, the supratherapeutic dose utilized in this study greatly exceeded exposure from any of these conditions.

The inclusion of only healthy subjects is consistent with guidance to minimize interfering variables. ${ }^{14}$ However, it is a limitation of this study as the drug is intended for use in patients with gout, many of whom are overweight and have cardiovascular disease. The use of a single lesinurad dose is also a potential limitation; however, lesinurad does not accumulate with repeat dosing. A positive aspect of this study design is the inclusion of both males and females.

\section{Conclusion}

Lesinurad, given at supratherapeutic doses up to $1,600 \mathrm{mg}$, does not cause QT interval prolongation or cardiac arrhythmia in healthy male or female subjects.

\section{Acknowledgments}

Funding was provided by Ardea Biosciences/AstraZeneca. Editorial support was provided by Tom Claus, PhD, of PAREXEL and funded by AstraZeneca. Ardea Biosciences, Inc. is a member of the AstraZeneca group.

\section{Disclosure}

Zancong Shen, Kathy Tieu, Mai Nguyen, Erin Harmon, David Wilson, and Caroline Lee are employees of Ardea Biosciences, Inc. Bradley Kerr is a paid consultant of Ardea Biosciences, Inc. Michael Gillen is an employee of AstraZeneca. The authors report no other conflicts of interest in this work.

\section{References}

1. Doghramji PP, Wortmann RL. Hyperuricemia and gout: new concepts in diagnosis and management. Postgrad Med. 2012;124(6):98-109.

2. Boss GR, Seegmiller JE. Hyperuricemia and gout. Classification, complications and management. N Engl J Med. 1979;300(26):1459-1468.

3. Neogi T. Clinical practice. Gout. N Engl J Med. 2011;364(5):443-452.

4. Khanna D, Fitzgerald JD, Khanna PP, et al; American College of Rheumatology. 2012 American College of Rheumatology guidelines for management of gout. Part 1: systematic nonpharmacologic and pharmacologic therapeutic approaches to hyperuricemia. Arthritis Care Res (Hoboken). 2012;64(10):1431-1446.

5. Richette P, Perez-Ruiz F, Doherty M, et al. Improving cardiovascular and renal outcomes in gout: what should we target? Nat Rev Rheumatol. 2014;10(11):654-661.

6. Zhang W, Doherty M, Bardin T, et al. EULAR evidence based recommendations for gout. Part II: management. Report of a task force of the EULAR Standing Committee for International Clinical Studies Including Therapeutics (ESCISIT). Ann Rheum Dis. 2006;65(10):1312-1324.

7. US Food and Drug Administration, HHS. International Conference on Harmonisation; guidance on E14 clinical evaluation of QT/QTc interval prolongation and proarrhythmic potential for non-antiarrhythmic drugs. Fed Regist. 2005;70(202):61134-61135.

8. Bobulescu IA, Moe OW. Renal transport of uric acid: evolving concepts and uncertainties. Adv Chronic Kidney Dis. 2012;19(6):358-371.

9. Fleischmann R, Kerr B, Yeh LT, et al. Pharmacodynamic, pharmacokinetic and tolerability evaluation of concomitant administration of lesinurad and febuxostat in gout patients with hyperuricaemia. Rheumatology (Oxford). 2014;53(12):2167-2174.

10. Girardet J-L, Miner JN. Urate crystal deposition disease and gout new therapies for an old problem. Annu Rep Med Chem. 2014;49: 151-164.

11. Garnett CE, Beasley N, Bhattaram VA, et al. Concentration-QT relationships play a key role in the evaluation of proarrhythmic risk during regulatory review. J Clin Pharmacol. 2008;48(1):13-18.

12. Bloomfield DM, Kost JT, Ghosh K, et al. The effect of moxifloxacin on QTc and implications for the design of thorough QT studies. Clin Pharmacol Ther. 2008;84(4):475-480.

13. Shah RR, Maison-Blanche P, Duvauchelle T, Robert P, Denis E. Establishing assay sensitivity in QT studies: experience with the use of moxifloxacin in an early phase clinical pharmacology study and comparison with its effect in a thorough QT study. Eur J Clin Pharmacol. 2015;71(12):1451-1459.

14. The International Council for Harmonisation [webpage on the Internet]. International Conference on Harmonization of Technical Requirements for Registration of Pharmaceuticals for Human Use. The Clinical Evaluation of QT/QTc Interval Prolongation and Proarrhythmic Potential for Non-Antiarrhythmic Drugs E-14. The International Council for Harmonisation. Available from: http://www.ich.org/products/guidelines/ efficacy/article/efficacyguidelines. Accessed April 4, 2016. 


\section{Publish your work in this journal}

Drug Design, Development and Therapy is an international, peerreviewed open-access journal that spans the spectrum of drug design and development through to clinical applications. Clinical outcomes, patient safety, and programs for the development and effective, safe, and sustained use of medicines are the features of the journal, which has also been accepted for indexing on PubMed Central. The manuscript management system is completely online and includes a very quick and fair peer-review system, which is all easy to use. Visit http://www.dovepress.com/testimonials.php to read real quotes from published authors.

Submit your manuscript here: http://www.dovepress.com/drug-design-development-and-therapy-journal 